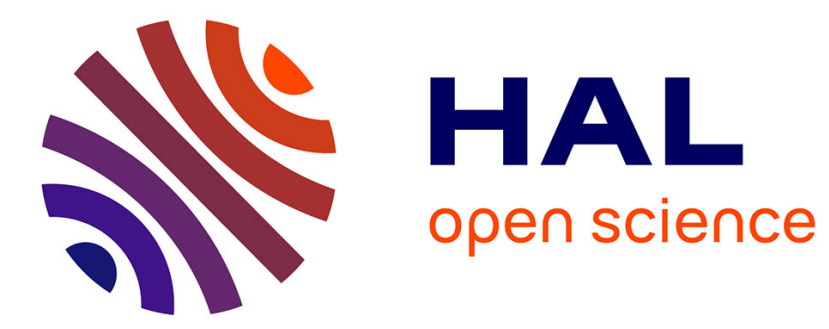

\title{
Photothérapie dynamique dans le cancer de la prostate à faible risque. Revue de la littérature
}

\author{
F. Kleinclauss, A. Frontczak, L. Balssa, S. Lebdai, R. Azzouzi
}

\section{To cite this version:}

F. Kleinclauss, A. Frontczak, L. Balssa, S. Lebdai, R. Azzouzi. Photothérapie dynamique dans le cancer de la prostate à faible risque. Revue de la littérature. Progrès en Urologie, 2019, 29, pp.393 401. 10.1016/j.purol.2019.05.004 . hal-03487977

\section{HAL Id: hal-03487977 \\ https://hal.science/hal-03487977}

Submitted on 20 Dec 2021

HAL is a multi-disciplinary open access archive for the deposit and dissemination of scientific research documents, whether they are published or not. The documents may come from teaching and research institutions in France or abroad, or from public or private research centers.
L'archive ouverte pluridisciplinaire HAL, est destinée au dépôt et à la diffusion de documents scientifiques de niveau recherche, publiés ou non, émanant des établissements d'enseignement et de recherche français ou étrangers, des laboratoires publics ou privés.

\section{(ㄷ)(1) $\$$}

Distributed under a Creative Commons Attribution - NonCommerciall 4.0 International 


\section{Photothérapie dynamique dans le cancer de la prostate à faible risque}

\section{Revue de la littérature}

François Kleinclauss $\S^{\S 1,2,3}$, Alexandre Frontczak ${ }^{\S^{1,2}}$, Loïc Balssa ${ }^{1,2}$, Souhil Lebdai ${ }^{4}$, Rahmene Azzouzi ${ }^{4}$

${ }^{1}$ Service d'Urologie, Andrologie et Transplantation Rénale, Centre Hospitalier Régional Universitaire, F-25000 Besançon, France

${ }^{2}$ Université Franche-Comté, F-25000 Besançon, France

${ }^{3}$ INSERM UMR 1098, F-25000 Besançon, France

${ }^{4}$ Service d'Urologie, Hôpital universitaire d'Angers, F-49933 Angers, France $\S$ contributed equally to this work

Adresse de correspondance

Pr François Kleinclauss (MD,PhD), Service d'Urologie-Andrologie et

Transplantation Rénale, CHRU Besançon

3 Bd A Fleming, 25000 Besançon, France

Tel : +33 381219170

Fax : +33 381219193

Mail : francois.kleinclauss@univ-fcomte.fr

\section{Déclaration d'intérêt :}


- Alexandre FRONTCZAK déclare ne pas avoir de conflits d'intérêts en lien avec cet article.

- Loïc BALSSA déclare ne pas avoir de conflits d'intérêts en lien avec cet article.

- François KLEINCLAUSS, déclare avoir été investigateur dans l'essai clinique PCM 301 et avoir été orateur rémunéré pour des symposiums organisés par STEBA Biotech. 


\section{Introduction :}

Le cancer de la prostate reste en France et dans les pays occidentaux le cancer le plus fréquent chez l'homme (48 427 nouveaux cas en 2013), et la troisième cause de décès par cancer (8625 décès en 2013) [1].

Du fait de l'utilisation plus large du dosage sanguin du PSA et de la détection précoce du cancer de prostate, l'incidence des cancers de faible risque selon la classification de d'AMICO a augmenté et représente actuellement de 40 à $50 \%$ des cancers diagnostiqués [2].

Les traitements dits curatifs comme la prostatectomie totale, la radiothérapie externe ou la curiethérapie restent les gold standard du traitement du cancer de la prostate localisé [3-5].

Si l'efficacité de ces traitements est démontrée dans les cancers de prostate à risque intermédiaires et à hauts risques, leur intérêt en survie globale ou survie spécifique dans le traitement des cancers à faibles risques a été récemment controversés [6-8]. De plus les effets secondaires connus de ces traitements comme l'incontinence urinaire, la dysfonction érectile et la toxicité digestive peuvent avoir des impacts majeurs sur la qualité de vie $[9,10]$. Une des options alternatives à ces traitements curatifs est la surveillance active (SA) développé par l'équipe de Toronto et Lawrence Klotz qui a démontré son intérêt dans les cancers de prostate à faible risque et à risque intermédiaire favorable [11]. Cependant à I'heure actuelle, seuls les cancers de la prostate à faible risque sont éligibles à une surveillance active [4]. Les critères de définition de ces cancers à faibles risques éligibles à une surveillance active sont multiples et principalement basés sur le PSA, le score de Gleason, le nombre de biopsies positives et les pourcentages d'envahissement tumoral. Les résultats des études de SA ont montré une excellente survie spécifique (98-100\%) avec un taux de traitement différé de 12 à 37\% [4]. Récemment l'actualisation des résultats de la cohorte de patients en SA de Toronto a montré une survie spécifique de $98 \%$ avec $68 \%$ des patients sans traitement 10 ans après le diagnostic [11].

Dans ce contexte, l'émergence des thérapies focales permettant le traitement exclusif de la tumeur et épargnant ainsi le tissu prostatique sain avoisinant apparaît comme une alternative séduisante [12]. L'objectif des traitements focaux serait ainsi le contrôle de la lésion tumorale au prix d'une morbidité minime. La thérapie focale est basée sur le concept du traitement de la lésion index, lésion de volume supérieur à $0,5 \mathrm{~cm}^{3}$ considérée comme la 
lésion principale et responsable de l'évolution et du pronostic du cancer de la prostate [13]. De même il a été démontré qu'en présence de lésions multifocales, la plupart des lésions secondaires présentait un volume et un grade plus faible que la lésion index expliquant ainsi que la multifocalité n’a jamais été démontré comme étant un facteur pronostic [14]. Liu et coll. ont aussi démontré que l'évolution métastatique du cancer de la prostate avait pour point de départ un seul clone cellulaire issu de la lésion index [15). Ainsi le concept de traitement de la seule lésion index, dit traitement focal, prend tout son sens. II permettrait la destruction de la lésion responsable de l'évolution locale et métastatique du cancer de prostate au prix d'une morbidité réduite [16]. Cependant, il a récemment été rapporté des cas de lésions métastatiques non issues de la lésion index ce qui a eu pour conséquence de raviver la controverse sur la théorie de la lésion index [17].

De nombreuses stratégies et techniques de thérapies focales ont été testées dans le traitement de différents cancers y compris le cancer de prostate [18, 19]. La photothérapie dynamique déjà utilisée en gastro-entérologie, dermatologie, ORL et gynécologie, fait partie de ces nouvelles technologies en cours de développement dans le cancer de la prostate.

\section{Méthode :}

Une revue systématique de la littérature de 1990 à 2017, par un groupe de travail de deux urologues cités comme auteurs, a été réalisé via les moteurs de recherche PubMed $^{\circledR}$ et EMBASE. Les mots clés utilisés ont été : " low-risk prostate cancer ", " focal treatment ", " vascular targeted photodynamic therapy " "TOOKAD ». Tous les articles en langue anglaise et française dont l'abstract est accessible ont été étudiés. Seuls les articles directement en rapport avec le sujet et ne constituant pas de doublons (mêmes résultats dans des revues différentes) ont été sélectionné. Au total, 394 publications référencées ont été analysées et 22 articles ont été retenus. (Figure 1).

\section{Résultats}




\section{Principes physiques de la photothérapie dynamique}

La première utilisation de la photothérapie dynamique remonte à plus d'un siècle par les observations de Raab, Tappeiner, et Jesionek [20, 21]. Le principe étant l'activation par une source de lumière d'un photosensibilisant qui devient alors cytotoxique pour le tissu environnant. Lors de la stimulation par une source de lumière à longueur d'onde propre à chaque photosensibilisant, celui-ci passe à un état d'excitation instable. Lors de son retour à un état stable, le photosensibilisant passe par un stade intermédiaire dit "état triplet » engendrant des réactions d'oxydoréduction conduisant à la formation de dérivés réactifs d'oxygène et d'azote (ROS et RNS) responsable de phénomènes d'apoptose et de nécrose cellulaires [22].

C'est en 1990 que fut réalisée la première utilisation de la photothérapie dynamique dans le cancer de la prostate localisé par Windhal et coll. Le photosensibilisant utilisé était un dérivé de l'hématoporphyrine, ayant une longue demi-vie d'action ( $>24 \mathrm{~h}$ ), exposant les patients à une photosensibilité cutanée prolongée [23]. Plus récemment, l'institut Weizmann (Rehovot, Israël) en collaboration avec le laboratoire STEBA Biotech (Luxembourg), a développé un nouveau type de photosensibilisant dérivé de la bacteriocholorophylle de palladium, la padoporfine (WST09) et plus récemment son dérivé hydrosoluble la padeliporfine (WST11, Tookad $^{\circledR}$ ) [24]. Ces molécules possèdent une demi-vie très courte, et leur activation n'est plus réalisée au niveau tissulaire mais en intravasculaire, d'où le terme de "vasculartargeted ". Le $\operatorname{Tookad}^{\circledR}$, agit par un triple mécanisme : physique, chimique et biologique. L'activation du WST11 par une lumière laser de longueur d'onde spécifique proche des rayons infra-rouge de $753 \mathrm{~nm}$ [25, 26] (mécanisme physique), réagit avec les molécules d'oxygène présente dans le sang, aboutissant à la formation de radicaux libres oxygénés (mécanisme chimique). Ces radicaux libres en grande quantité vont interagir avec les cellules endothéliales vasculaires et aboutir à une occlusion vasculaire (mécanisme biologique) (Figure 2). Cette occlusion vasculaire est présente dans tout le territoire "illuminé » et entraine ainsi une nécrose ischémique de la zone traitée et donc une ischémie tumorale (Figure 3). Pour la VTP prostatique, le photosensibilsant WST 11 est délivré par voie systémique intraveineuse. L'illumination est réalisée de manière homogène par une ou plusieurs sources LASER introduites dans la prostate par l'intermédiaire de fibres optiques 
insérées dans des cathéters transparents posés par voie transpérinéale sous contrôle échographique.

La procédure de VTP par TOOKAD ${ }^{\circ}$ a été mise au point initialement par Trachtenberg en 2004 [27, 28, 29] puis diffusée en Europe par Emberton et Azzouzi [30, 31]. Elle a depuis été largement utilisée et standardisée lors des essais de phase I/II et III de la photothérapie dynamique à visée vasculaire par WST09 et WST11 [27] (Figure 4).

\section{Résultats des essais cliniques}

Les premières études cliniques évaluant la photothérapie dynamique ont été réalisées en traitement de rattrapage après radiothérapie externe chez des patients atteints d'un cancer de la prostate de faible risque localisé [28, 29, 32]. En 2007, Trachtenberg et coll. [28] ont publié un essai de phase I, visant à étudier les paramètres pharmacocinétiques du WST09 et la dose d'illumination nécessaire à l'ablation de la tumeur cible. Les résultats étaient évalués par la présence de nécrose prostatique en IRM à 7 jours et par l'absence de cellules tumorales sur biopsies prostatiques à 6 mois accompagnées d'une seconde IRM. Les meilleurs résultats étaient obtenus à la dose maximale de $2 \mathrm{mg} / \mathrm{kg}$ de WSTO9 et une illumination de $200 \mathrm{mWatts} / \mathrm{cm}$. Les biopsies réalisées au sein des zones traitées retrouvaient des zones acellulaires hyalinisées et fibrosées sans signes d'adénocarcinome. Les biopsies positives se retrouvaient toujours en dehors de ces zones traitées. L'étude des effets indésirables n'a pas retrouvé de phénomènes de photosensibilité, ni d'altération de la fonction érectile ou mictionnelle. Les évènements indésirables rapportés étaient principalement des hypotensions (environ $50 \%$ des patients) lors de l'administration du photosensibilisant, hypotension rapportée à l'utilisation du cremophor comme excipient solubilisant du WST09.

L'essai suivant de phase II avait pour but de traiter l'ensemble de la glande prostatique avec une dose de WSTO9 de $2 \mathrm{mg} / \mathrm{kg}$ chez des patients en échec de radiothérapie prostatique. L'étude a montré $61 \%$ de biopsies négatives à 6 mois que lorsque $90 \%$ du volume prostatique recevait une dose de lumière supérieure à $230 \mathrm{~J} / \mathrm{cm} 2$. Malheureusement, deux cas de fistules recto-urétrales ont été rapportés, potentiellement en lien avec des lésions radiques du rectum préexistantes à la VTP [29]. 
Après élaboration du WST11, dérivé hydrosoluble du WST09, limitant ainsi les effets d'hypotension lors de son administration [24], trois essais de phase II ont été publiés [30, 31, 33] (tableau 1). Leur but était d'évaluer la dose optimale, la faisabilité et la tolérance de la photothérapie dynamique à visée vasculaire (VTP) par WST11 dans la prise en charge initiale de patients atteints de cancer de la prostate localisé de faible risque.

Les critères d'inclusion étaient les hommes > 18 ans avec un cancer de la prostate jusqu'à un stade clinique T2b-NO-MO (mais incluant les stades radiologiques et anatomopathologiques T2c), un taux de PSA < 10ng/ml. En cas de biopsies écho-guidées par voie endorectale, la limite du score de Gleason était de 3+3, contrairement au diagnostic par biopsies périnéales ou un stade 4 minoritaire était toléré (si $<3$ biopsies positives par lobe et une longueur maximum de $3 \mathrm{~mm}$ par biopsie). Les critères d'exclusions étaient : tout traitement antérieur ou actuel, y compris les manipulations hormonales ou déprivation androgénique dans les 6 mois. Le suivi comportait une IRM une semaine après la procédure et ainsi qu'une IRM et des biopsies 6 mois après la procédure de VTP. Le taux de PSA était évalué à 1, 3 et 6 mois.

Le critère de jugement principal était défini par le résultat des biopsies prostatiques à 6 mois, le succès étant défini par des biopsies négatives dans le lobe traité. Les critères de jugement secondaire étaient le taux de PSA à 1, 3, 6 mois, et le pourcentage de nécrose des volumes cibles sur l'IRM à 7 jours et 6 mois

L'étude PCM 201, incluant 40 patients, avait pour but de définir la posologie et la dose de lumière optimales pour l'obtention de biopsies négatives à 6 mois [30]. II a ainsi été montré qu'un traitement optimal avec $4 \mathrm{mg} / \mathrm{kg}$ de WST11 et une dose de lumière de $200 \mathrm{~J} / \mathrm{cm}^{2}$ permettait l'obtention $55 \%$ des biopsies négatives à 6 mois. Une relation linéaire entre l'énergie de lumière reçue et le volume cible traité, défini par le LDI (Light Density Index) a aussi été retrouvée. Le LDI apparaît comme un critère majeur de succès de la procédure. Ainsi, en cas de LDI> à 1, le nombre de patients avec des biopsies négatives à 6 mois passait à $83 \%$ alors qu'il n'était que de $31 \%$ en cas de $L D I<$ à $1(p<0.01)$. Les mêmes résultats ont été observés sur le volume de nécrose. Le pourcentage moyen de volume de tissus nécrosé/volume de tissus cible était de $95 \%$ quand le $L D I>1$, contre seulement $59 \%$ en cas de LDI $<1(p<0.01)$.

Ces résultats ont été confirmés par l'étude européenne de phase II, PCM 203, incluant 85 patients [31]. Les pourcentages de biopsies négatives et de nécrose du volume cible étaient de $82,6 \%$ et de $87,6 \%$ en cas de traitement optimum. Plus récemment une analyse 
" poolée » des résultats des 3 études de phase 2 a montré que sur les 117 patients traités par $4 \mathrm{mg} / \mathrm{kg}$ de WST11 et avec une dose de lumière de 200J/cm, 68,4\% (IC 95\% 59,1-76, 8\%) avaient des biopsies négatives à 6 mois et un pourcentage de nécrose du volume cible sur I'IRM à 7 jours de 76,5\% [33]. Lors de l'analyse en sous-groupe des patients ayant reçu un traitement optimal avec un LDI > 1, 77,6\% (IC 95\% 66,6-86,4\%) présentaient des biopsies négatives à 6 mois avec un pourcentage moyen de nécrose du volume cible de $86,3 \%$. Le taux de PSA montrait une décroissance moyenne d'environ 2,0ng/ml à 3 et 6 mois [33].

Un des centres participants aux 3 études Européennes de phase 2 a publié en 2017 le suivi prospectif de l'ensemble des 82 patients traités avec un suivi médian de 68 mois. Les auteurs rapportent que $82 \%$ des patients traités par PTD n'avaient plus de cancer cliniquement significatif dans les lobes traités et $76 \%$ des patients avaient ainsi pu éviter un traitement radical durant le temps de l'étude [34].

Le retentissement et la morbidité du traitement sur les fonctions urinaires et érectiles ont également été évalués grâce aux scores de qualité de vie IPSS et IIEF-5, établi avant VTP puis à 1,3 et 6 mois. Concernant le statut mictionnel, les 3 études ont des résultats concordants avec une légère détérioration du score IPPS à 1 mois post-procédure puis une amélioration à 6 mois (diminution moyenne du score IPSS de -1,8) [33]. Ces résultats restaient stables avec un suivi de médian de 68 mois [34].

L'hypothèse émise est l'impact de la procédure (ponction prostatique périnéal et nécrose ischémique aigue de la prostate) entrainant par un mécanisme inflammatoire une augmentation du volume prostatique. Passée la phase aigu, la nécrose ischémique entraine une atrophie du lobe traité, objectivé par l'IRM entrainant ainsi une amélioration de l'IPSS par rapport à l'IPSS pré-traitement [35]. La qualité de vie urinaire évaluée par l'IPSS montrait une amélioration 6 mois après traitement.

L'impact de la VTP sur la dysfonction érectile a aussi été évalué notamment par le score IIEF5. La baisse moyenne du score IIEF à 6 mois était de 3 points dans l'analyse poolée sans que l'impact clinique puisse être analysé [33].

Les différents évènements indésirables rapportés au Medical Dictionary of Regulatory Activities ont été évalués. Sur l'ensemble des 3 études de phase II [33], 82,9\% des patients ont au moins présenté un événement indésirable enregistré. Ils étaient majoritairement considérés comme légers (grade 1 ou 2). Les évènements les plus rapportés étaient la 
dysurie $(33,3 \%)$, la dysfonction érectile $(16,2 \%)$, les douleurs périnéales $(15,4 \%)$, l’hématurie $(13,7 \%)$, la rétention aigüe d'urine $(11,1 \%)$ ou les urgenturies $(9,4 \%) .9,4 \%$ (11 patients) des patients ont présenté des événement indésirables sérieux (> Grade 3), 3 d'entre eux n'ont pas été mis en lien avec l'étude ou la procédure (un cas de neuropathie ischémique optique, un cas de gastrite, et un cas d'ulcère gastro-duodénal avec hématémèse). Deux ont été mis en relation avec une large nécrose extra-prostatique en raison d'une déviation de la procédure. Les autres étaient en lien avec des douleurs périnéales, une hématurie, ou une prostatite. Au cours de ces essais, aucun événement indésirable n'a entrainé de sortie d'étude. Aucun évènement indésirable n'a entrainé de ré-intervention ou de défaillance organique vitale. Selon la classification de morbidité chirurgicale de Clavien-Dindo aucune complication classée IV ou V n'a été reportée [36]. Aucun cas de fistule uretro-rectale n'a été rapporté sur l'ensemble des 3 essais de phase II. L'analyse des données de I'IRM postopératoire (réalisé 7 jours après VTP) montrait une nécrose extra-prostatique chez 67,2\% des patients, non symptomatique la plupart du temps et non associée à des événements cliniques significatifs [35].

L'analyse histologique des biopsies de contrôles des zones prostatiques traitées par VTP lors des essais de phase II a montré des lésions de fibroses avec hypercollagénose et dépôts hyalins remplaçant le tissu prostatique glandulaire [37]. Cette fibrose s'accompagnait d'ilots de nécrose ischémique. La capsule apparaissait intacte ou légèrement fibreuse. La zone de traitement était toujours à distance de la capsule (résultats attendus du fait de la procédure). II est intéressant de noter que toutes les lésions d'adénocarcinome retrouvée après VTP ont été retrouvée dans des zones de traitements incomplets (le plus souvent à proximité de la capsule prostatique). Aucune lésion d'adénocarcinome n'a été retrouvée dans les zones cicatricielles de fibrose ou de nécrose prostatique post VTP. Ces données confirment l'efficacité de la photothérapie dans le contrôle histologique de la maladie et posent la question des marges de sécurités vis à vis de la capsule prostatique à adopter pour permettre un contrôle optimum du cancer.

Suite à ces essais cliniques de phase II, l'évaluation de la VTP dans le cancer de prostate localisé s'est poursuivi par la mise en œuvre d'un essai multicentrique européen prospectif randomisé de phase III comparant les résultats entre la VTP avec TOOKAD (WST11) et la 
surveillance active dans le cadre de la prise en charge du cancer de la prostate de très faible risque. (NCT01310894) [38].

Cette étude a inclus 413 patients au sein de 47 centres européens avec un suivi de 24 mois (tableau 2). Les critères d'inclusions étaient similaires à ceux déterminés pour l'éligibilité à la surveillance active (limité au gleason 3, 3 biopsies maximum positives, longueur tumorale entre 3 et $5 \mathrm{~mm}$, stade clinique limité jusqu'au pT2a). L'évaluation a été réalisée par mesure du PSA et toucher rectal tous les 3 mois, ainsi que des biopsies prostatiques par voie transrectale à 12 et 24 mois. Les critères de jugement principaux étaient l'absence de cancer à 24 mois définit par l'histologie des biopsies prostatiques, et l'échec du traitement défini par la progression histologique vers un cancer de risque modéré ou de haut risque nécessitant une prise en charge thérapeutique.

Cette étude a montré que les patients traités par VTP avaient un meilleur taux de biopsies négatives à 24 mois que les patients en surveillance active $(R R=3.67$, IC 95\% 2.53-5.33, $\mathrm{p}<0,0001)$. La VTP était associée à un moindre risque de progression du cancer (HR 0.34, IC $95 \%$ 0.24-0.46, $\mathrm{P}<0.0001$ ), et à une durée avant progression plus longue ( 28.3 mois, IC $95 \%$ 26.0-30.6) que pour la surveillance active (14.1 mois, IC 95\% 12.9-23.8) ( $p<0.0001)$ [38].

Comme dans les essais de phase II, le statut mictionnel et la fonction érectile ont été évaluées par les scores IPSS et IIEF-5. II a été montré une détérioration transitoire initiale dans le cadre de la VTP, sans différence entre les 2 groupes à 24 mois pour le statut mictionnel et érectile. De plus l'évaluation de la qualité de vie par le score EQ-5d a montré que celle-ci n'était pas différente entre les 2 groupes $(p=0.64)$.

L'ensemble des évènements indésirables reportés pour les patients traités par VTP sont essentiellement modérés et transitoire avec au premier plan des évènements de la sphère génito-urinaires (rétention aigüe d'urine, hématurie, dysurie, douleur périnéale) [38].

\section{Discussion}


La VTP a démontré sa faisabilité et sa reproductibilité dans le traitement des cancers de prostate à faible risque avec une bonne tolérance clinique, la majorité des évènements indésirables étant modérés et transitoires, et ayant peu d'impact sur la fonction érectile et urinaire. Cette bonne tolérance clinique semble similaire aux autres types de thérapies focales, comme l'ont montré Barret et coll. [39].

L'utilisation de la VTP, avec une dose optimale de WST11 de $4 \mathrm{mg} / \mathrm{kg}$ et d'une dose de lumière de $200 \mathrm{~J} / \mathrm{cm}$ à $753 \mathrm{~nm}$ avec un LDI > 1 semblent prometteur avec un taux de biopsies prostatiques négatives dans le lobe traité à 6 mois d'environ 80\% [40]. Cependant la réalisation des biopsies sous contrôle échographique par voie endorectale peut entrainer un biais notamment en terme de reproductibilité avec un risque d'erreur estimé à 20-25\% [41]. Le développement d'outil comme les plateformes de fusion IRM/Echographie et la réalisation de biopsies ciblées devraient permettre à l'avenir d'améliorer la fiabilité et la reproductibilité des biopsies [42].

Une des critiques généralement faites envers les traitements focaux est la problématique de l'échec de cette prise en charge, et du traitement de sauvetage. Dans le cas de la VTP, l'étude des traitements de rattrapage par prostatectomie totale après échec d'une prise en charge par VTP a été réalisée par Lebdai et coll. [43]. Malgré le petit effectif de la cohorte (19 patients), les auteurs ont montré que celle-ci était faisable et efficace. Environ $80 \%$ des patients ont eu des suites opératoires standard, avec un taux de continence de $68 \%$ en postopératoire. II n'a pas été rapporté de blessures rectales durant les interventions, et il n'y a pas eu de laparo-conversion lors des procédures de laparoscopie standard ou robot-assistée. La difficulté de certaines interventions a été liée au caractère bilatéral de la VTP pour un adénocarcinome touchant les deux lobes, en raison de la fibrose péri-prostatique engendrée. Dans ce cas le risque de marges positives était plus important ( $R R=4,3, p=0,003)$, comme le risque de difficulté per-opératoire $(R R=5,4, p=0,009)$. De même, le taux de marge positive dans cette étude était corrélé au caractère bilatéral de la VTP. Ces difficultés opératoires et ces résultats sont cependant à modérer. Les patients de cette série sont issus des études de phase II comprenant une analyse de l'effet-dose. Certains patients ont donc eu une VTP non optimale. De plus ces difficultés n'ont pas été retrouvées lors des PT après VTP d'un seul lobe et devrait à fortiori être encore minimisées en cas de traitement focal pur.

Comparé aux autres thérapies focales, tel que l'HIFU, la VTP a des résultats similaires. Dans 
une revue de la littérature de Warmuth et coll. [44], les hommes traités par HIFU avaient un taux de biopsies négatives à 3 mois de $86 \%$. Rischmann et coll. [45], dans une série prospective de 111 patients traités par HIFU, avaient retrouvé un taux de biopsies sans cancer cliniquement significatif à un an de $95 \%$.

Cependant à l'heure actuelle, il n'existe pas de données comparatives entre les différentes modalités de thérapies focales.

\section{Conclusion :}

La photothérapie dynamique à visée vasculaire avec le WST11 (Tookad) apparaît comme une alternative intéressante dans la prise en charge du cancer de la prostate localisé de faible risque, permettant un contrôle satisfaisant du cancer de prostate au prix d'une morbidité faible et sans effets indésirables graves. Cette thérapeutique, comme tous les traitements focaux, suscite aujourd'hui un vif intérêt dans le cancer de la prostate comme en témoigne le nombre d'essais en cours recensé $(>70)$ [46]. Cependant de nombreuses questions restent encore aujourd'hui sans réponse comme sa place dans l'arsenal thérapeutique, ses indications, les critères de succès, la surveillance après traitement [47]. Ce traitement, comme tous les traitements focaux reste actuellement dans le domaine de la recherche et ne peut être proposé en dehors d'essais thérapeutiques à des patients informés et minutieusement sélectionnés $[4,5]$.

\section{Références:}

1. Le cancer de la prostate - Les cancers les plus fréquents | Institut National Du Cancer http://www.e-cancer.fr/Professionnels-de-sante/Les-chiffres-du-cancer-enFrance/Epidemiologie-des-cancers/Les-cancers-les-plus-frequents/Cancer-de-laprostate

2. Cooperberg MR, Moul JW, Carroll PR. The changing face of prostate cancer. J Clin Oncol Off J Am Soc Clin Oncol. 2005;23(32):8146 51. 
3. Rébillard X, Grosclaude P, Leone N, Velten M, Coureau G, Villers A, et al. [Incidence and mortality of urological cancers in 2012 in France]. Progres En Urol J Assoc Francaise Urol Soc Francaise Urol. 2013;23 Suppl 2:S57-65.

4. Rozet F, Hennequin C, Beauval J-B, Beuzeboc P, Cormier L, Fromont G, et al. Recommandations en onco-urologie 2016-2018 du CCAFU : Cancer de la prostate. Prog En Urol. 2016;27:S95 143.

5. Mottet N, Bellmunt J, Bolla M, Briers E, Cumberbatch MG, De Santis M, et al. EAUESTRO-SIOG Guidelines on Prostate Cancer. Part 1: Screening, Diagnosis, and Local Treatment with Curative Intent. Eur Urol. 2017;71(4):618 29.

6. Wilt TJ, Brawer MK, Jones KM, Barry MJ, Aronson WJ, Fox S, et al. Radical Prostatectomy versus Observation for Localized Prostate Cancer. N Engl J Med. 2012;367(3):203 13.

7. Bill-Axelson A, Holmberg L, Ruutu M, Garmo H, Stark JR, Busch C, et al. Radical prostatectomy versus watchful waiting in early prostate cancer. N Engl J Med. 2011;364(18):1708 17.

8. Hamdy FC, Donovan JL, Lane JA, Mason M, Metcalfe C, Holding P, et al. 10-Year Outcomes after Monitoring, Surgery, or Radiotherapy for Localized Prostate Cancer. N Engl J Med. 2016;375(15):1415 24.

9. Resnick MJ, Koyama T, Fan K-H, Albertsen PC, Goodman M, Hamilton AS, et al. LongTerm Functional Outcomes after Treatment for Localized Prostate Cancer. N Engl J Med. 2013;368(5):436 45.

10. Donovan JL, Hamdy FC, Lane JA, Mason M, Metcalfe C, Walsh E, et al. Patient-Reported Outcomes after Monitoring, Surgery, or Radiotherapy for Prostate Cancer. N Engl J Med. 2016;375(15):1425 37.

11. Klotz L, Vesprini D, Sethukavalan P, Jethava V, Zhang L, Jain S, et al. Long-Term FollowUp of a Large Active Surveillance Cohort of Patients With Prostate Cancer. J Clin Oncol. 2015;33(3):272 7 .

12. Emberton M. Why Focal Therapy is a Legitimate and Necessary Response to a Changing World. J Urol. 2015;194(4):875 6.

13. Ahmed HU. The index lesion and the origin of prostate cancer. $\mathrm{N}$ Engl J Med. 2009;361(17):1704 6 .

14. Bott SRJ, Ahmed HU, Hindley RG, Abdul-Rahman A, Freeman A, Emberton M. The index lesion and focal therapy: an analysis of the pathological characteristics of prostate cancer. BJU Int. 2010;106(11):1607 11.

15. Liu W, Laitinen S, Khan S, Vihinen M, Kowalski J, Yu G, et al. Copy number analysis indicates monoclonal origin of lethal metastatic prostate cancer. Nat Med. 2009;15(5):559 65. 
16. Lecornet E, Ahmed HU, Moore CM, Emberton M. Conceptual basis for focal therapy in prostate cancer. J Endourol. 2010;24(5):811 8.

17. Haffner MC, Mosbruger T, Esopi DM, Fedor H, Heaphy CM, Walker DA, et al. Tracking the clonal origin of lethal prostate cancer. J Clin Invest. 2013;123(11):4918 22.

18. Ahmed HU, Moore C, Emberton M. Minimally-invasive technologies in uro-oncology: the role of cryotherapy, HIFU and photodynamic therapy in whole gland and focal therapy of localised prostate cancer. Surg Oncol. 2009;18(3):219 32.

19. Valerio M, Cerantola Y, Eggener SE, Lepor H, Polascik TJ, Villers A, et al. New and Established Technology in Focal Ablation of the Prostate: A Systematic Review. Eur Urol. 2017;71(1):17 34 .

20. Raab O. Uber die Wirkung fluoreszierden Stoffen.

21. Tappeiner $\mathrm{H}$, Jesionek A. Therapeutische Versuchi mit fluoreszierender.

22. Colin P, Estevez J-P, Betrouni N, Ouzzane A, Puech P, Leroy $X$, et al. Thérapie photodynamique et cancer de la prostate. Prog Urol. 2011;21(2):85-92.

23. Windahl T, Andersson SO, Lofgren L. Photodynamic therapy of localised prostatic cancer. Lancet Lond Engl. 1990;336(8723):1139.

24. Ashur I, Goldschmidt R, Pinkas I, Salomon Y, Szewczyk G, Sarna T, et al. Photocatalytic generation of oxygen radicals by the water-soluble bacteriochlorophyll derivative WST11, noncovalently bound to serum albumin. J Phys Chem A. 2009;113(28):8027 37.

25. Betrouni N, Lopes R, Puech P, Colin P, Mordon S. A model to estimate the outcome of prostate cancer photodynamic therapy with TOOKAD Soluble WST11. Phys Med Biol. 2011;56(15):4771 83.

26. Moore CM, Mosse CA, Allen C, Payne H, Emberton M, Bown SG. Light penetration in the human prostate: a whole prostate clinical study at $763 \mathrm{~nm}$. J Biomed Opt. 2011;16(1):015003.

27. Azzouzi A-R, Lebdai S, Benzaghou F, Stief C. Vascular-targeted photodynamic therapy with TOOKAD ${ }^{\circledR}$ Soluble in localized prostate cancer: standardization of the procedure. World J Urol. 2015;33(7):937 44.

28. Trachtenberg J, Bogaards A, Weersink RA, Haider MA, Evans A, McCluskey SA, et al. Vascular targeted photodynamic therapy with palladium-bacteriopheophorbide photosensitizer for recurrent prostate cancer following definitive radiation therapy: assessment of safety and treatment response. J Urol. 2007;178(5):1974 1979.

29. Trachtenberg J, Weersink RA, Davidson SRH, Haider MA, Bogaards A, Gertner MR, et al. Vascular-targeted photodynamic therapy (padoporfin, WST09) for recurrent prostate cancer after failure of external beam radiotherapy: a study of escalating light doses. BJU Int. 2008;102(5):556 62. 
30. Moore CM, Azzouzi A-R, Barret E, Villers A, Muir GH, Barber NJ, et al. Determination of optimal drug dose and light dose index to achieve minimally invasive focal ablation of localised prostate cancer using WST11-vascular-targeted photodynamic (VTP) therapy. BJU Int. 2015;116(6):888 96.

31. Azzouzi A-R, Barret E, Moore CM, Villers A, Allen C, Scherz A, et al. TOOKAD ${ }^{\circledR}$ Soluble vascular-targeted photodynamic (VTP) therapy: determination of optimal treatment conditions and assessment of effects in patients with localised prostate cancer. BJU Int. 2013;112(6):766 74 .

32. Lepor H. Vascular targeted photodynamic therapy for localized prostate cancer. Rev Urol. 2008;10(4):254 61.

33. Azzouzi AR, Barret E, Bennet J, Moore C, Taneja S, Muir G, et al. TOOKAD ${ }^{\circledR}$ Soluble focal therapy: pooled analysis of three phase II studies assessing the minimally invasive ablation of localized prostate cancer. World J Urol. 2015;33(7):945-53.

34. Lebdai S, Bigot P, Leroux P-A, Berthelot L-P, Maulaz P, Azzouzi A-R. Vascular Targeted Photodynamic Therapy with Padeliporfin for Low Risk Prostate Cancer Treatment: Midterm Oncologic Outcomes. J Urol. 2017;198(2):335 44.

35. Kulik M, Nedelcu C, Martin F, Lebdai S, Rousselet MC, Azzouzi AR, et al. Post-treatment MRI aspects of photodynamic therapy for prostate cancer. Insights Imaging. 2014;5(6):697 713.

36. Dindo D, Demartines N, Clavien P-A. Classification of surgical complications: a new proposal with evaluation in a cohort of 6336 patients and results of a survey. Ann Surg. 2004;240(2):205 13.

37. Eymerit-Morin C, Zidane M, Lebdai S, Triau S, Azzouzi AR, Rousselet M-C. Histopathology of prostate tissue after vascular-targeted photodynamic therapy for localized prostate cancer. Virchows Arch Int J Pathol. 2013;463(4):547 52.

38. Azzouzi A-R, Vincendeau S, Barret E, Cicco A, Kleinclauss F, van der Poel HG, et al. Padeliporfin vascular-targeted photodynamic therapy versus active surveillance in men with low-risk prostate cancer (CLIN1001 PCM301): an open-label, phase 3, randomised controlled trial. Lancet Oncol. 2017;18(2):181 91.

39. Barret E, Ahallal Y, Sanchez-Salas R, Galiano M, Cosset J-M, Validire P, et al. Morbidity of focal therapy in the treatment of localized prostate cancer. Eur Urol. 2013;63(4):618 22.

40. Coleman J, Scherz A. Focal therapy of localised prostate cancer by vascular targeted photodynamic therapy with WST-11 (TOOKAD ${ }^{\circledR}$ Soluble). Eur Urol Rev. 2012;7(2):106 8 . 
41. Berglund RK, Masterson TA, Vora KC, Eggener SE, Eastham JA, Guillonneau BD. Pathological upgrading and up staging with immediate repeat biopsy in patients eligible for active surveillance. J Urol. 2008;180(5):1964-1967.

42. Schoots IG, Roobol MJ, Nieboer D, Bangma CH, Steyerberg EW, Hunink MGM. Magnetic resonance imaging-targeted biopsy may enhance the diagnostic accuracy of significant prostate cancer detection compared to standard transrectal ultrasound-guided biopsy: a systematic review and meta-analysis. Eur Urol. 2015;68(3):438 50.

43. Lebdai S, Villers A, Barret E, Nedelcu C, Bigot P, Azzouzi A-R. Feasibility, safety, and efficacy of salvage radical prostatectomy after Tookad ${ }^{\circledR}$ Soluble focal treatment for localized prostate cancer. World J Urol. 2015;33(7):965 71.

44. Warmuth M, Johansson T, Mad P (2010) Systematic review of the efficacy and safety of high-intensity focussed ultrasound for the primary and salvage treatment of prostate cancer. Eur Urol. 2010;58:803-815.

45. Rischmann P, Gelet A, Riche B, et al (2016) Focal High Intensity Focused Ultrasound of Unilateral Localized Prostate cancer: A Prospective Multicentric Hemiablation Study of 111 Patients. Eur Urol. 2016;71:267-73.

46. Baydoun A, Traughber B, Morris N, Abi Zeid Daou M, McGraw M, Podder TK, et al. Outcomes and toxicities in patients treated with definitive focal therapy for primary prostate cancer: systematic review. Future Oncol. 2016;13(7):649 63.

47. de la Rosette J, Ahmed H, Barentsz J, Johansen TB, Brausi M, Emberton M, et al. Focal therapy in prostate cancer-report from a consensus panel. J Endourol.

2010;24(5):775 80.

\section{Légendes des figures:}

Figure 1. Flow chart de la sélection des articles, Flow chart for the selection of the articles

Figure2. Triple mécanisme d'action de VTP, Triple mechanism of action of VTP

Figure 3. Images IRM en coupe axiale séquence T2, avant traitement (a) et à 6 mois post VTP (b) [35], Axial T2-weighted MR images from before treatment (a) and 6 months after treatment (b) [35] 
Figure 4. Procédure opératoire : ponction transpérinéale et installation des fibres laser, Procédure : transperineal puncture and introduction of laser fibers

Tableau 1. Caractéristiques des critères d'inclusion, des modalités de traitement et des principaux résultats des essais cliniques de phase II, Characteristics of inclusion criteria, treatment modalities and main results of phase II clinical trials

Tableau 2. Caractéristiques des critères d'inclusion, des modalités de traitement, de suivie et des principaux résultats de l'essais clinique de phase III PCM 301, Characteristics of inclusion criteria, treatment and monitoring modalities, and main results of phase III clinical trial PCM 301 
Recherche dans PubMed et Embase

"Low-risk prostate cancer »

" Focal treatment »

"Vascular targeted photodynamic therapy »

" TOOKAD »

$N=394$

Titres et abstracts exclus

Motifs : langue, thème principale non

concordant

$\mathbf{N}=351$

Articles sélectionnés

$N=43$

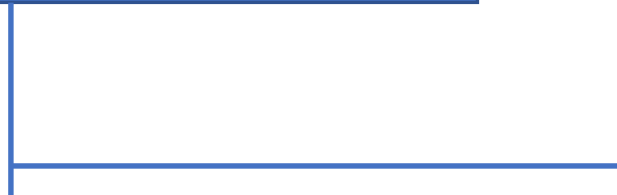

Articles exclus :

Motifs : résultats redondants

$\mathbf{N}=\mathbf{2 1}$

Articles retenus

$N=22$

Figure 1. Flow chart de la sélection des articles 


\section{Mécanisme d'action VTP :}

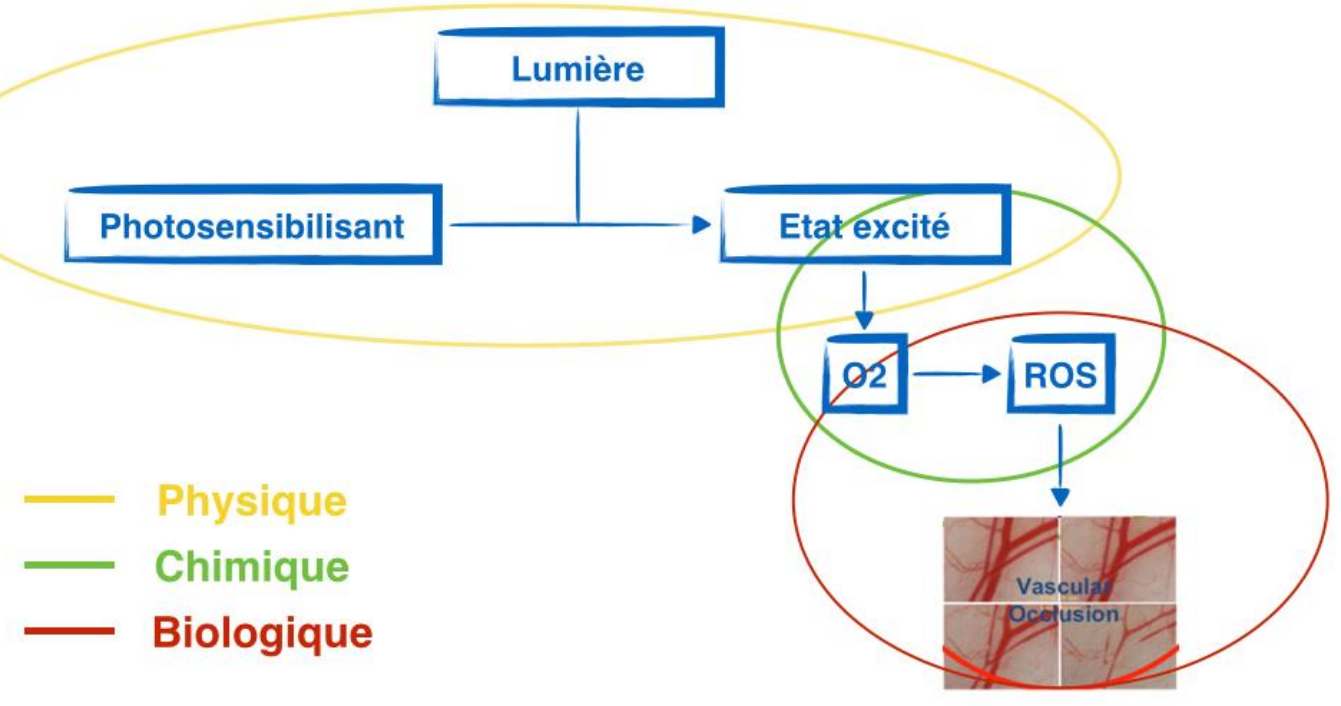

Figure 2. Triple mécanisme d'action de VTP 


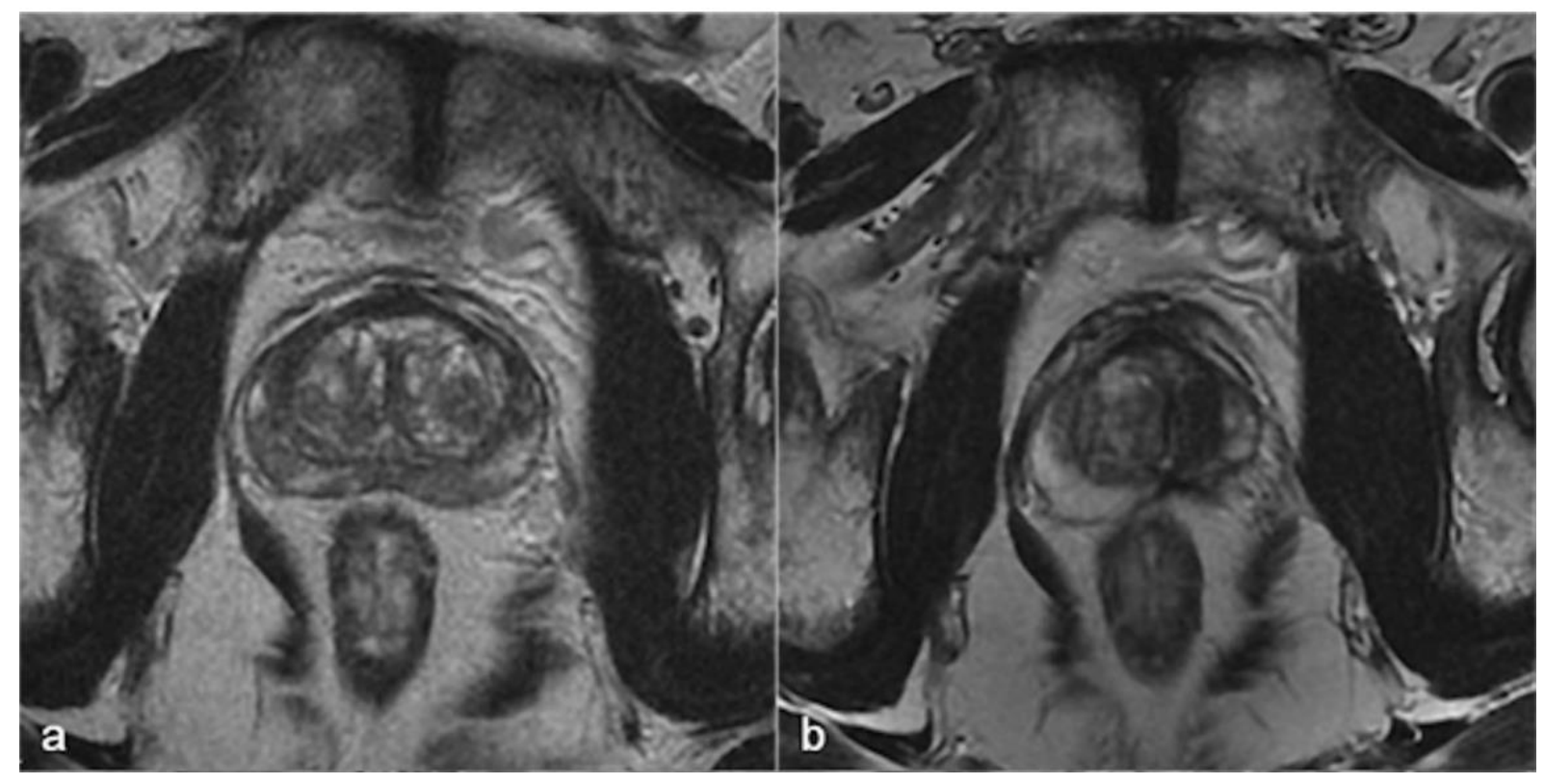

Figure 3. Images IRM en coupe axiale séquence T2, avant traitement (a) et à 6 mois post VTP (b) [35] 


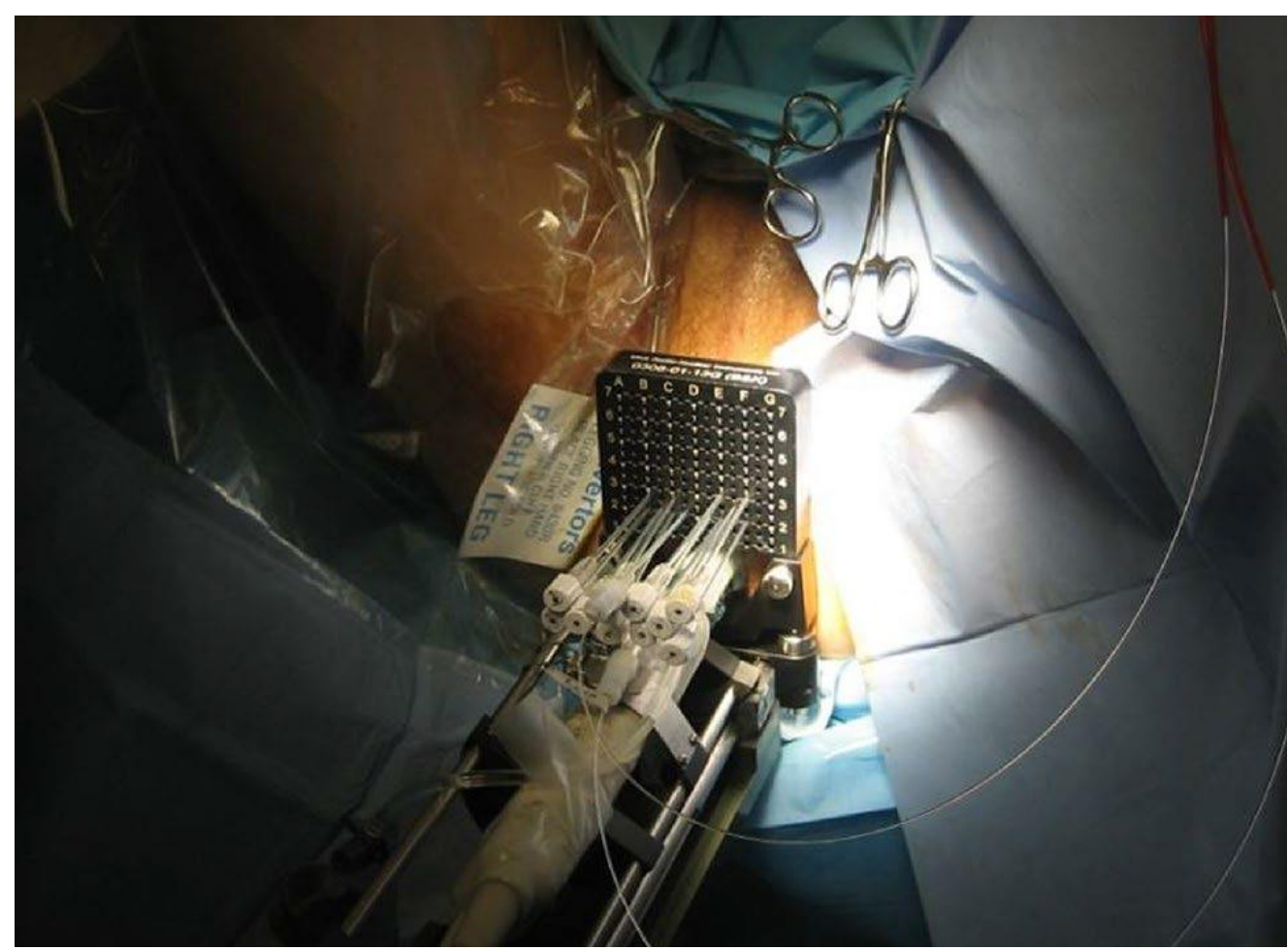

Figure 4. Procédure opératoire : ponction transpérinéale et installation des fibres laser. 
Tableau 1. Caractéristiques des critères d'inclusions, des modalités de traitement et des principaux résultats des essais cliniques de phase II

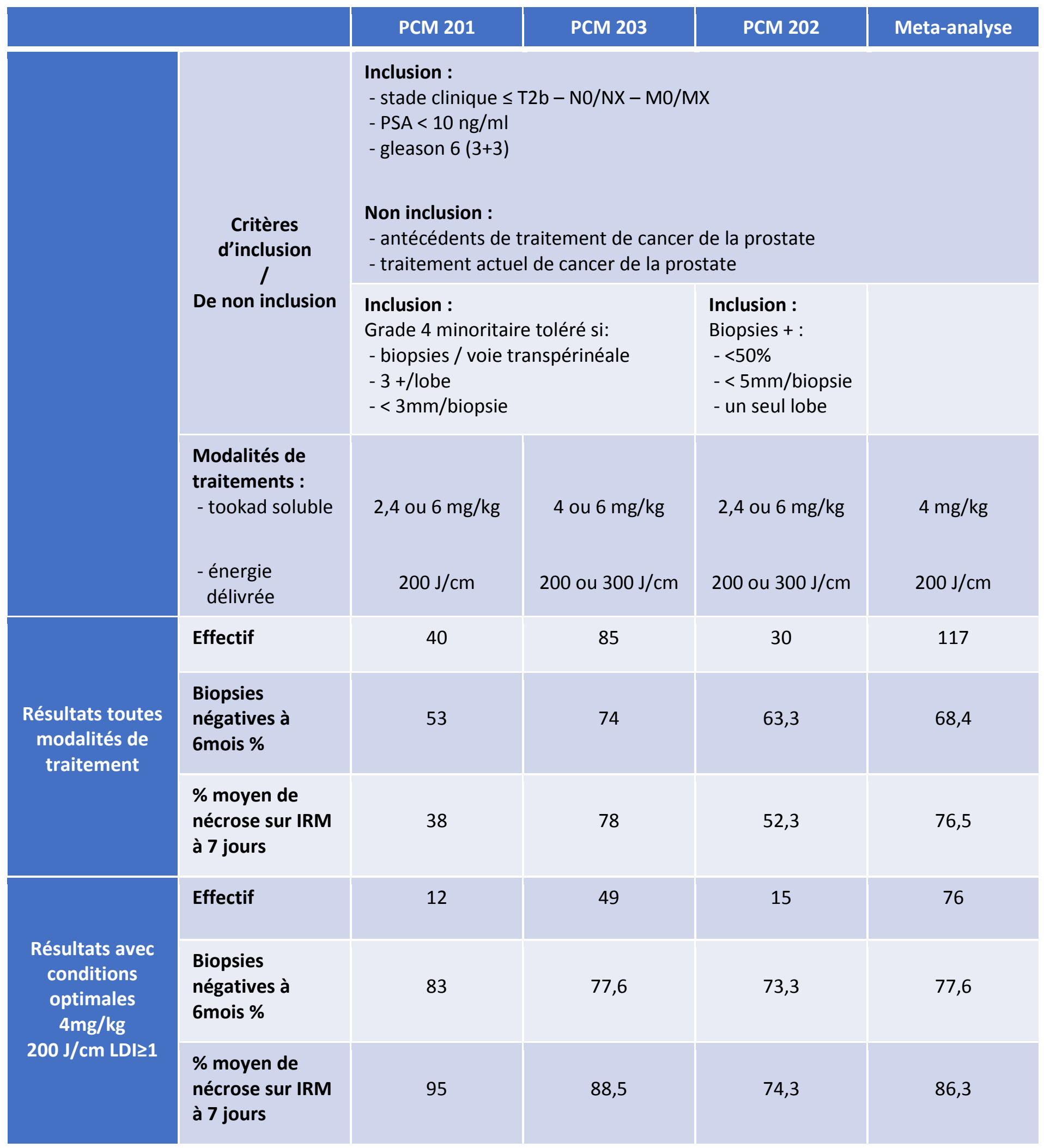


Tableau2. Caractéristiques des critères d'inclusion, des modalités de traitement, de suivi et des résultats principaux de l'essais clinique de phase III PCM 301

\begin{tabular}{|c|c|c|}
\hline & VTP & Surveillance active \\
\hline Critères d'inclusion & \multicolumn{2}{|c|}{$\begin{array}{c}\text { Gleason } 6(3+3) \\
\text { PSA }<10 \\
\text { Stade clinique jusqu'à T2a } \\
\text { Si } 1 \text { biopsie }+ \text { : longueur tumorale } \geq 3 \mathrm{~mm} \text { et } \leq 5 \mathrm{~mm} \\
\text { Si plusieurs biopsies + : longueur tumorale / biopsie } \leq 5 \mathrm{~mm}\end{array}$} \\
\hline Modalités de traitement & $\begin{array}{l}\text { Tookad soluble : } 4 \mathrm{mg} / \mathrm{kg} \\
\text { Énergie délivrée } 200 \mathrm{~J} / \mathrm{cm} \\
\text { LDI } \geq 1\end{array}$ & \\
\hline Suivi & \multicolumn{2}{|c|}{$\begin{array}{l}\text { PSA et toucher rectal tous les } 3 \text { mois } \\
\text { Biopsies de prostate à } 12 \text { et } 24 \text { mois }\end{array}$} \\
\hline Effectif & 206 & 207 \\
\hline Biopsies négatives à 24 mois \% & \multicolumn{2}{|c|}{$H R=3,67$ IC $95 \%(2,53-5,33) p<0,0001$} \\
\hline Progression à 24 mois \% & \multicolumn{2}{|c|}{$H R=0,34$ IC $95 \%(0,24-0,46) p<0,001$} \\
\hline
\end{tabular}

